\title{
Novel Schiff bases derived from 5-aminobarbituric acid: synthesis and solid state structure
}

\author{
Ina Bolz, Claudia May, and Stefan Spange* \\ "Department of Polymer Chemistry, Institute of Chemistry, Chemnitz University of Technology, \\ Straße der Nationen 62, D-09111 Chemnitz, Germany \\ E-mail: stefan.spange@,chemie.tu-chemnitz.de
}

\section{Dedicated to Ernst Anders on the occasion of his $65^{\text {th }}$ birthday}

\begin{abstract}
Synthesis and characterization of novel Schiff bases with multiple binding sites for supramolecular assemblies are presented. For this purpose 1,3-dimethyl- and 1-butyl-5aminobarbituric acid $(\mathbf{1} \mathbf{a}, \mathbf{1 b})$ are condensed with para-nitro- and para- $N, N$-dimethylaminocinnamaldehyde, respectively. Solid state structures of 5-[(1E,2E)-3-(4-nitrophenyl)- and 5[(1E,2E)-3-\{4-(dimethylamino)phenyl\}prop-2-enyliden]-prop-2-enyliden] aminobarbituric acid derivatives (2a, 2b, 3a, 3b) have been investigated by means of solid state ${ }^{13} \mathrm{C}\left\{{ }^{1} \mathrm{H}\right\}$ NMR- and FTIR-spectroscopy. Analyses of the spectra information suggest that in the solid state the Schiff bases exist only in the enol form.
\end{abstract}

Keywords: Dyes/pigments, barbituric acid, Schiff bases, NMR spectroscopy

\section{Introduction}

Artificial receptors for biologically active molecules have attracted attention from the viewpoint of molecular recognition. ${ }^{[1]}$ Because derivatives of barbituric acid are important members of the pyrimidine family, they are suitable for this purpose. But their biological activity is mainly attributed to tautomerism, acid-base equilibriums, and to the nature of their substituents. For example, many scientists in recent years have shown, that related Merocyanine dyes are effective sensitizers with interesting photophysical properties. ${ }^{[2,3]}$

In this communication we report on synthesis and solid state structure of Schiff bases derived from 5-aminobarbituric acid (uramil) derivatives and para-substituted cinnamaldehydes (Scheme $1)$. 


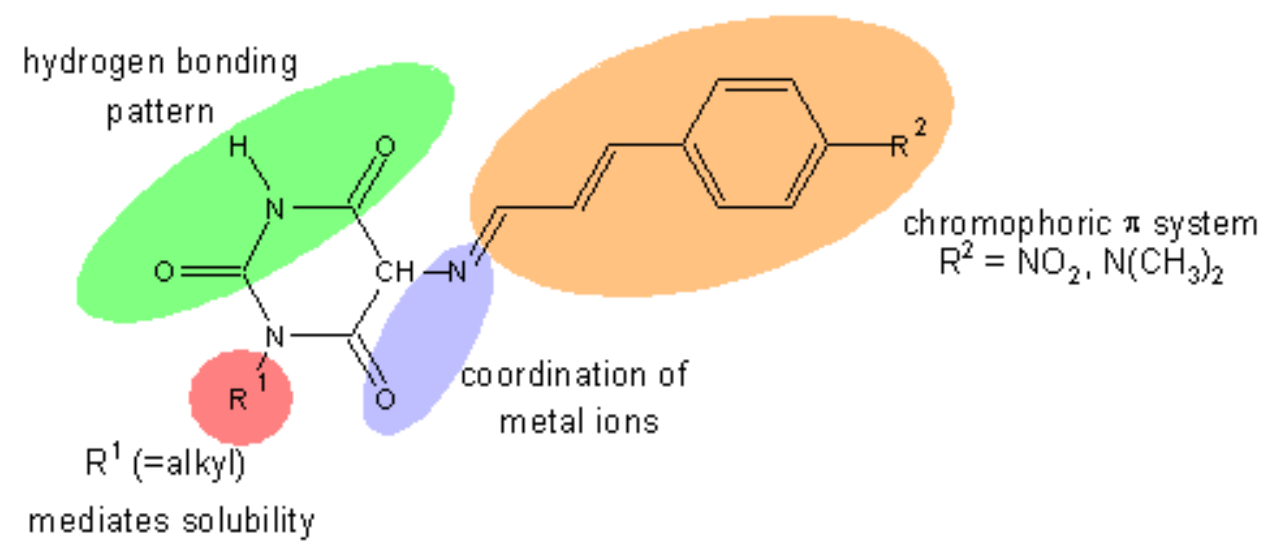

Scheme 1. Chemical structure and functionalities of the target Schiff bases.

We chose those solvatochromic organic chromophores with hydrogen bonding functions, because of their potential to bind reversibly to metal ions and/or complementary bases such as 2,6-diaminopyridine. ${ }^{[4-7]}$ Furthermore, the 5-aminobarbituric acid unit can be substituted at one of the ring nitrogen atoms, which is suitable to adjust the solubility of the compound under retention of the ADA sequence $(\mathrm{A}=$ hydrogen bond acceptor site corresponds to the $\mathrm{CO}$ group, $\mathrm{D}=$ hydrogen bond donor site corresponds to the $\mathrm{NH}$ group). However, the enol form of this type of compounds appears to be more stable than the keto form which is an objective of this work.

\section{Results and Discussion}

According to the related literature, ${ }^{[8]}$ the class of compounds to be investigated (Scheme 2) should be readily accessible by condensation reaction of 5-aminobarbituric acids with aromatic aldehydes. Carrying out the synthesis, however, was not trivial. Many experiments which were performed in alcoholic solvents using literature procedures, failed due the isolation problems. The reactants and desired products are poorly soluble despite the introduction of $N$-alkyl groups. 4-Nitrocinnamaldehyde and 4-( $N, N$-dimethylamino)cinnamaldehyde were finally chosen, because the Schiff base adducts of other aldehydes, i. e. derived from 4-nitrobenzaldehyde or 4pyridinaldehyde, form strong hydrogen bonded complexes with the reactant uramil. 


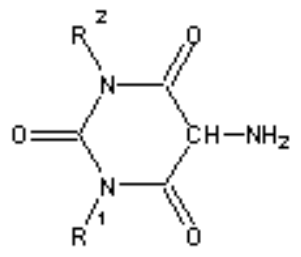

Uramil derivatives

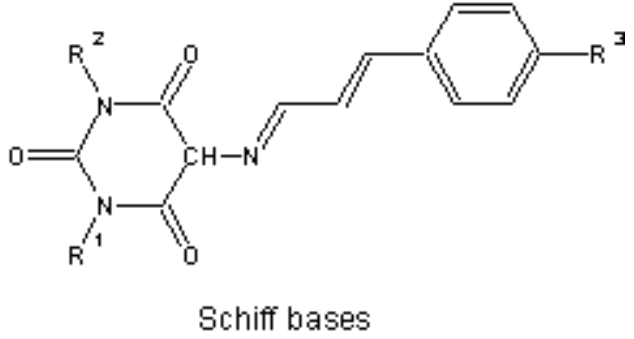

\begin{tabular}{cccc}
\hline & $\begin{array}{c}\text { Uramil } \\
\text { derivative }\end{array}$ & $\begin{array}{c}\text { Schiff base } \\
\mathrm{R}^{3}=\mathrm{NO}_{2}\end{array}$ & $\begin{array}{c}\text { Schiff base } \\
\mathrm{R}^{3}=\mathrm{N}\left(\mathrm{CH}_{3}\right)_{2}\end{array}$ \\
\hline $\mathrm{R}^{1}=\mathrm{R}^{2}=\mathrm{H}$ & $\mathbf{1}$ & $\mathbf{( 2 )}$ & $\mathbf{( 3 )}$ \\
$\mathrm{R}^{1}=\mathrm{R}^{2}=\mathrm{CH}_{3}$ & $\mathbf{1 a}$ & $\mathbf{2 a}$ & $\mathbf{3 a}$ \\
$\mathrm{R}^{1}=\mathrm{H}, \mathrm{R}^{2}=n-\mathrm{C}_{4} \mathrm{H}_{9}$ & $\mathbf{1 b}$ & $\mathbf{2 b}$ & $\mathbf{3 b}$ \\
\hline
\end{tabular}

Scheme 2. Novel solvatochromic compounds and their reactants studied in this work. Syntheses of dyes in parentheses were not successful due to strong adduct formation between reactant and product.

Although uramil and its derivatives are well known since a long time, however there are no data relating specifically to their solid state structure. ${ }^{[9,11-14]}$ The mostly published keto structure is not tenable. The absence of the keto form in uramil derivatives is indicated by IR spectroscopy. Because no absorption bands for the $\mathrm{NH}_{2}$ stretch vibration were found, Ivin et al discussed the presence of a zwitterionic ammonium enolate of some $\mathrm{N}$-methylderivatives of uramil. ${ }^{[9]}$ We confirmed this structure for the compounds $\mathbf{1}$, $\mathbf{1 a}$ and $\mathbf{1 b}$ by FTIR spectroscopy measured in diffuse reflection. These uramil derivatives have bipolar structures with the positive charge situated on the exocyclic nitrogen atom and the negative charge on the system of the ring (Scheme 3).

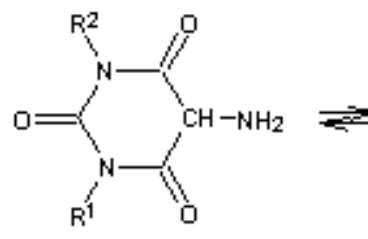

keto

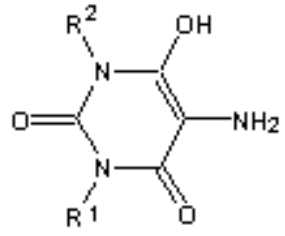

enol

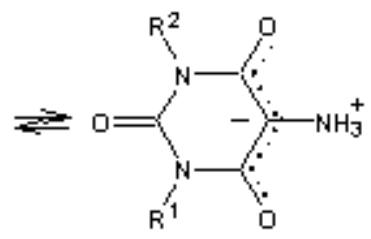

enolate

Scheme 3. The zwitterionic ammonium enolate structure of the uramil derivatives 1, $\mathbf{1 a}$ and $\mathbf{1 b}$, confirmed by FTIR spectroscopy.

1: $\mathrm{R}^{1}=\mathrm{R}^{2}=\mathrm{H}$

1a: $\mathrm{F}^{1}=\mathrm{F}^{2}=\mathrm{CH}_{3}$

1b: $\mathrm{R}^{1}=H_{1} \mathrm{R}^{2}=\mathrm{RC}_{4} \mathrm{H}_{\mathrm{g}}$ 
For the structural characterization of the novel Schiff bases and their starting compounds ${ }^{13} \mathrm{C}\left\{{ }^{1} \mathrm{H}\right\}$ CP/MAS solid state NMR spectroscopy has been used in particular, because high resolution solution state ${ }^{13} \mathrm{C}$ NMR spectroscopy was not suitable due to the poor solubility.
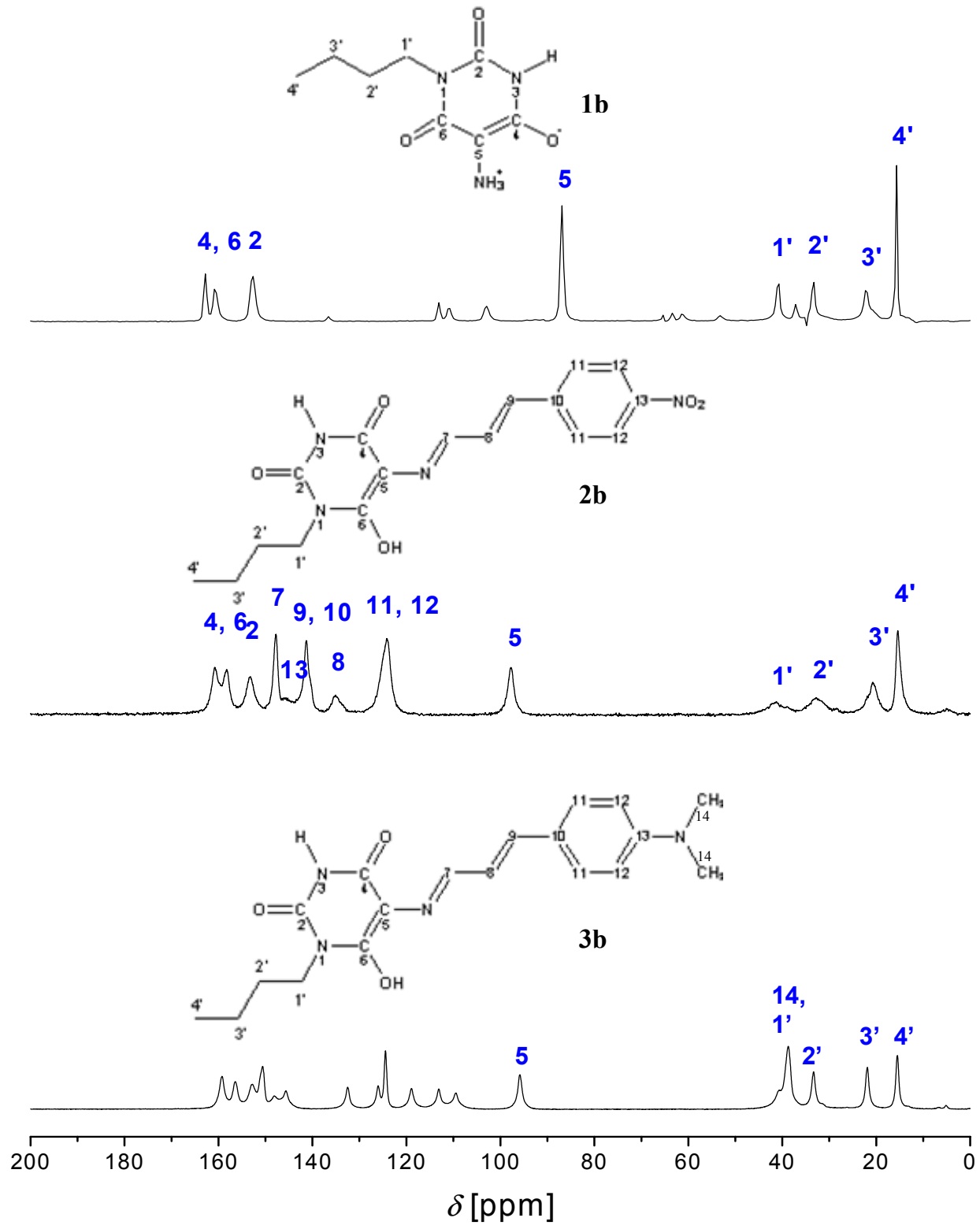

Figure 2. ${ }^{13} \mathrm{C}\left\{{ }^{1} \mathrm{H}\right\}$ CP/MAS solid state NMR spectra of 1-butyl-5-aminobarbituric acid 1b, 1butyl-5-[(1E,2E)-3-\{4-nitrophenyl\}prop-2-enyliden]amino barbituric acid 2b, and 1-butyl-5[(1E,2E)-3-\{4-(dimethylamino)phenyl\} prop-2-enyliden]aminobarbituric acid $\mathbf{3 b}$, recorded at MAS frequencies of $5 \mathrm{kHz}(\mathbf{1 b})$ and $12 \mathrm{kHz}(\mathbf{2 b}, \mathbf{3 b})$. Unassigned peaks are spinning sidebands, with exception of spectrum of $\mathbf{3 b}$. 
Notably, the absence of an aliphatic $\mathrm{CH}$ proton peak in the ${ }^{1} \mathrm{H}$ NMR spectra as well as the missing carbon peak at about $55 \mathrm{ppm}$ in the corresponding ${ }^{13} \mathrm{C}\left\{{ }^{1} \mathrm{H}\right\} \quad \mathrm{CP} / \mathrm{MAS}$ spectra unambiguously confirm an enol structure for all investigated Schiff bases (Figure 2). The enolic solid state structure of the $N, N$-dimethylamino-substituted barbituric acids $\mathbf{3 a}$ and $\mathbf{3 b}$ is nontrivial. In this dyes a push-push $\pi$-electron system is present. Likely, different bipolar structures as suggested in Scheme 3 contribute. Therefore, a clear assignment of all peaks to a structure element is not possible on basis of available data. Solely, the strong downfield shift of the carbon C-5 rules out the keto form.
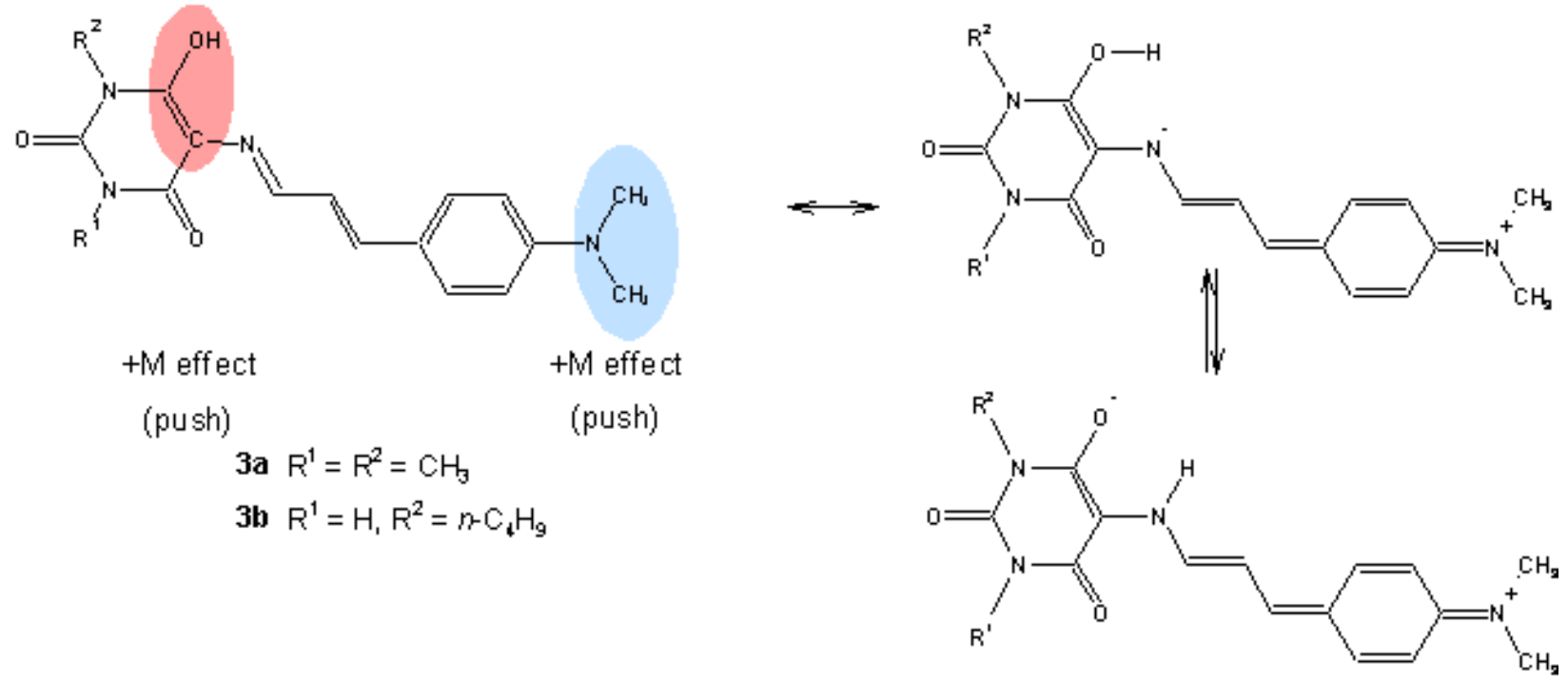

Scheme 3. Suggested bipolar character of the $N, N$-dimethylamino-substituted barbituric acids 3a and $\mathbf{3 b}$.

\section{Experimental Section}

General Procedures. Unless otherwise noted, all materials were used as received from a commercial supplier without further purification. All reaction solvents were redistilled over appropriate drying agents prior to use. Melting points were taken on a micro melting point apparatus and are uncorrected. Elemental analyses were recorded on a Vario EL (Elementar Analysensysteme GmbH Hanau). FTIR spectra were measured with a BIO-RAD FTS 165 spectrometer in diffuse reflection with $\mathrm{KBr}$ as reference. The intensity is indicated as follows: $\mathrm{s}$ (strong), $\mathrm{m}$ (medium), w (weak) and b (broad). ${ }^{[10]} \mathrm{All}{ }^{1} \mathrm{H}$ and ${ }^{13} \mathrm{C}$ NMR spectra were obtained either on a Varian Gemini-300 spectrometer or on a Bruker Avance 250 spectrometer. Chemical shifts were reported as $\delta$-values in parts per million $(\mathrm{ppm})$ relative to $\mathrm{Si}\left(\mathrm{CH}_{3}\right)_{4}$ as relative reference $(\delta=0 \mathrm{ppm})$ and to the solvent as internal reference. Coupling constants $(J)$ were reported in Hertz $(\mathrm{Hz})$. Peak multiplicities are indicated as follows: $\mathrm{s}$ (singlet), $\mathrm{d}$ (doublet), $\mathrm{t}$ 
(triplet), q (quartet), quin (quintet), se (sextet), m (multiplet) and b (broad). High resolution solid state ${ }^{13} \mathrm{C}$ NMR spectra were recorded at $100 \mathrm{MHz}$ on a Bruker Avance 400 digital spectrometer using cross polarization (CP) and standard Bruker magic-angle spinning (MAS) probes with zirkonia rotors (4 $\mathrm{mm}$ and $7 \mathrm{~mm}$ external diameters). Different MAS frequencies $(5-12 \mathrm{kHz})$ were used. Chemical shifts were given relative to $\mathrm{Si}\left(\mathrm{CH}_{3}\right)_{4}$, established using adamantane as an external reference.

General Procedures. Syntheses of barbituric acid and 5-amino-barbituric acid derivatives are described in the literature. ${ }^{[11-14]}$ However, no NMR data have been reported for 1-butyl barbituric acid and 1-butyl-5-aminobarbituric acid $\mathbf{1 b}$.

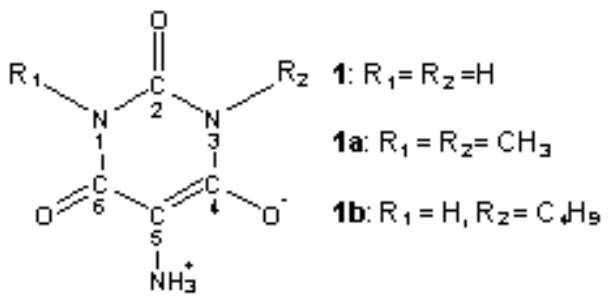

Synthesis of the Schiff bases. The 5-aminobarbituric acid derivative ( $2.92 \mathrm{mmol})$ is dissolved in $100 \mathrm{ml}$ dried ethanol and added to a solution of the aldehyde compound $(2.92 \mathrm{mmol})$ in $50 \mathrm{ml}$ dried ethanol. The mixture is refluxed for $4 \mathrm{~h}$. Then, the reaction vessel is stored overnight and the coloured precipitate is filtered off, washed with ethanol and dried.

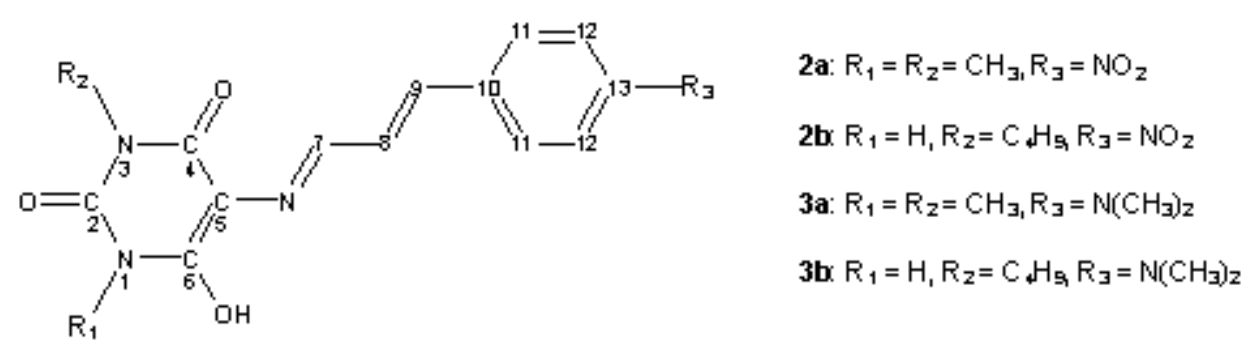

\section{Compound Characterization}

1-Butylbarbituric acid. White solid; m.p.: $109-110{ }^{\circ} \mathrm{C} ;{ }^{1} \mathrm{H}$ NMR $\left(300 \mathrm{MHz}, \mathrm{CDCl}_{3}\right): \delta 0.91(\mathrm{t}$, $\left.{ }^{3} J_{4^{\prime} 3^{\prime}}=7.3 \mathrm{~Hz}, 3 \mathrm{H}, \mathrm{CH}_{3}\right), 1.32\left(\mathrm{se}, 2 \mathrm{H}, \mathrm{CH}_{2}\right), 1.54$ (quin, $\left.2 \mathrm{H}, \mathrm{CH}_{2}\right), 3.63(\mathrm{~s}, 2 \mathrm{H}, 5), 3.82\left(\mathrm{t},{ }^{3} J_{1}{ }^{\prime} 2^{\prime}\right.$ $\left.=7.3 \mathrm{~Hz}, 2 \mathrm{H}, \mathrm{N}-\mathrm{CH}_{2}\right), 9.39(\mathrm{~s}, 1 \mathrm{H}, 3):{ }^{13} \mathrm{C} \mathrm{NMR}\left(75 \mathrm{MHz}, \mathrm{CDCl}_{3}\right): \delta 13.59\left(\mathrm{CH}_{3}\right), 19.90\left(\mathrm{CH}_{2}\right)$, $29.84\left(\mathrm{CH}_{2}\right), 39.19\left(\mathrm{~N}-\mathrm{CH}_{2}\right), 41.12(5), 150.79$ (2), 165.31 (4/6), 165.38 (4/6).

5-Aminobarbituric acid (1). White solid; yield: 82\%; m.p.: decomp. $>300{ }^{\circ} \mathrm{C}$; IR (cm $\left.{ }^{-1}\right): 3084$ (m), 1725 (m), 1710 (m), 1582 (s), 1544 (s), 1536 (s), 1501 (m), $1413(\mathrm{~s}) ;{ }^{13} \mathrm{C}\left\{{ }^{1} \mathrm{H}\right\}$ CP MAS NMR (4 mm, $12 \mathrm{kHz}$ ): $\delta 86.78(5), 153.92(2), 162.78(4,6)$; anal. calculated for $\mathrm{C}_{4} \mathrm{H}_{5} \mathrm{~N}_{3} \mathrm{O}_{3}$ (found): C 33.57 (33.67), H 3.52 (3.42), N 29.37 (29.32). 
1,3-Dimethyl-5-aminobarbituric acid (1a). White solid, yield: 46\%; m.p.: decomp. $>200{ }^{\circ} \mathrm{C}$; IR ( $\left.\mathrm{cm}^{-1}\right)$ : 3049 (m), 2980 (m), 1714 (s), 1636 (m), 1594 (w), 1571 (m), 1482 (m), 1459 (m); ${ }^{13} \mathrm{C}\left\{{ }^{1} \mathrm{H}\right\}$ CP MAS NMR (4 mm, $\left.12 \mathrm{kHz}\right): \delta 30.10\left(\mathrm{~N}-\mathrm{CH}_{3}\right), 84.46(5), 151.20(2), 160.32(4,6)$; anal. calculated for $\mathrm{C}_{6} \mathrm{H}_{9} \mathrm{~N}_{3} \mathrm{O}_{3}$ (found): $\mathrm{C} 42.10$ (42.00), $\mathrm{H} 5.30$ (5.14), $\mathrm{N} 24.55$ (24.55).

1-Butyl-5-aminobarbituric acid (1b). White solid, yield: 38\%; m.p.: decomp. $>200{ }^{\circ} \mathrm{C}$; IR $\left(\mathrm{cm}^{-1}\right): 3188(\mathrm{w}), 3107(\mathrm{w}), 3018(\mathrm{~m}), 2960,2875(\mathrm{~m}), 1690,1679,1663$ (s), $1629(\mathrm{~m}), 1613(\mathrm{~s})$, $1501(\mathrm{~s}), 1455(\mathrm{~s}) ;{ }^{13} \mathrm{C}$ CP MAS NMR $(7 \mathrm{~mm}, 5 \mathrm{kHz}): \delta 15.56\left(\mathrm{CH}_{3}\right), 22.15\left(\mathrm{CH}_{2}\right), 33.40\left(\mathrm{CH}_{2}\right)$, $40.80\left(\mathrm{~N}-\mathrm{CH}_{2}\right), 87.01$ (5), 152.83 (2), 160.89 (4/6), 162.75 (4/6); anal. calculated for $\mathrm{C}_{8} \mathrm{H}_{13} \mathrm{~N}_{3} \mathrm{O}_{3}$ (found): C 48.23 (47.52), H 6.58 (6.41), N 21.09 (20.64).

1,3-Dimethyl-5-[(1E,2E)-3-(4-nitrophenyl)prop-2-enyliden]aminobarbituric acid (2a). Red solid; yield: 90\%; m.p.: 237-239 ${ }^{\circ} \mathrm{C}$; IR $\left(\mathrm{cm}^{-1}\right)$ : 3099 (m), 3049 (m), 2999 (m), 1698 (s), 1633,

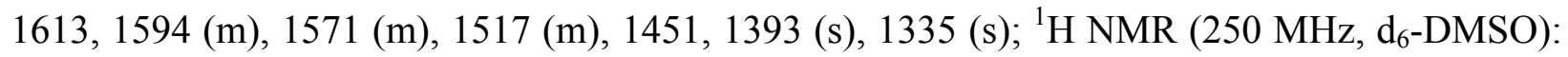
$\delta 3.16\left(\mathrm{~s}, 6 \mathrm{H}, \mathrm{N}-\mathrm{CH}_{3}\right), 7.49\left(\mathrm{~d},{ }^{3} J_{9,8}=15.6 \mathrm{~Hz}, 1 \mathrm{H}, 9\right), 7.77\left(\mathrm{~d},{ }^{3} J_{11,12}=8.9 \mathrm{~Hz}, 2 \mathrm{H}, 11\right), 7.86(\mathrm{dd}$, $\left.{ }^{3} J_{8,9}=15.6 \mathrm{~Hz},{ }^{3} J_{8,7}=10.3 \mathrm{~Hz}, 1 \mathrm{H}, 8\right), 8.27\left(\mathrm{~d},{ }^{3} J_{12,11}=8.9 \mathrm{~Hz}, 2 \mathrm{H}, 12\right), 9.55\left(\mathrm{~d},{ }^{3} J_{7,8}=10.3 \mathrm{~Hz}\right.$, $1 \mathrm{H}, 7) ;{ }^{13} \mathrm{C}\left\{{ }^{1} \mathrm{H}\right\}$ CP MAS NMR $(7 \mathrm{~mm}, 5 \mathrm{kHz}): \delta 28.09\left(\mathrm{~N}-\mathrm{CH}_{3}\right), 30.45\left(\mathrm{~N}-\mathrm{CH}_{3}\right), 98.50(5)$, 124.68 (12), 128.63 (11), 131.58 (8), 141.89 (9, 10), 145.86 (13), 148.42 (7) 151.08 (2), 159.15 $(4,6)$; anal. calculated for $\mathrm{C}_{15} \mathrm{H}_{14} \mathrm{~N}_{4} \mathrm{O}_{5}$ (found): C 54.54 (54.29), H 4.27 (4.18), N 16.96 (16.82).

1-Butyl-5-[(1E,2E)-3-(4-nitrophenyl)prop-2-enyliden]aminobarbituric acid (2b). Red solid; yield: 78\%; m.p.: $277-279{ }^{\circ} \mathrm{C}$; IR ( $\left.\mathrm{cm}^{-1}\right)$ : 3165 (m), $3076(\mathrm{w}), 3026$ (m), 2960, 2875 (m), 1690, 1660 (s), 1609, 1594 (m), 1575 (m), 1517 (m), 1451, 1428 (s), 1339 (s); ${ }^{1} \mathrm{H}$ NMR (250 MHz, d $6^{-}$ DMSO): $\delta 0.90\left(\mathrm{t},{ }^{3} J_{4}, 3^{\prime}=7.1 \mathrm{~Hz}, 3 \mathrm{H}, \mathrm{CH}_{3}\right) 1.28\left(\mathrm{~m}, 2 \mathrm{H}, \mathrm{CH}_{2}\right), 1.49\left(\mathrm{~m}, 2 \mathrm{H}, \mathrm{CH}_{2}\right), 3.74\left(\mathrm{t},{ }^{3} J_{1}\right.$, , $2^{\prime}$ $\left.=7.1 \mathrm{~Hz}, 2 \mathrm{H}, \mathrm{N}-\mathrm{CH}_{2}\right), 7.32\left(\mathrm{~d},{ }^{3} J_{9,8}=15.5 \mathrm{~Hz}, 1 \mathrm{H}, 9\right), 7.69\left(\mathrm{dd},{ }^{3} J_{8,9}=15.5 \mathrm{~Hz},{ }^{3} J_{8,7}=10.1 \mathrm{~Hz}\right.$, $1 \mathrm{H}, 8), 7.77\left(\mathrm{~d},{ }^{3} J_{11,12}=8.9 \mathrm{~Hz}, 2 \mathrm{H}, 11\right), 8.27\left(\mathrm{~d},{ }^{3} J_{12,11}=8.9 \mathrm{~Hz}, 2 \mathrm{H}, 12\right), 9.53\left(\mathrm{~d},{ }^{3} J_{7,8}=10.1\right.$ $\mathrm{Hz}, 1 \mathrm{H}, 7), 10.39(\mathrm{~s}, 1 \mathrm{H}, 3) ;{ }^{13} \mathrm{C}\left\{{ }^{1} \mathrm{H}\right\}$ CP MAS NMR (4 mm, $\left.12 \mathrm{kHz}\right): \delta 15.43\left(\mathrm{CH}_{3}\right), 20.75$ $\left(\mathrm{CH}_{2}\right), 32.83\left(\mathrm{CH}_{2}\right), 41.29\left(\mathrm{~N}_{-} \mathrm{CH}_{2}\right), 97.76$ (5), 124.16 (11, 12), 135.09 (8), 141.30 (9, 10), 145.89 (13), 147.79 (7) 153.25 (2), 158.26 (4/6), 160.75 (4/6); anal. calculated for $\mathrm{C}_{17} \mathrm{H}_{18} \mathrm{~N}_{4} \mathrm{O}_{5}$ (found): C 56.98 (56.42), H 5.06 (5.01), N 15.64 (15.29).

\section{1,3-Dimethyl-5-[(1E,2E)-3-\{4-(dimethylamino)phenyl\}prop-2-enyliden]-aminobarbituric}

acid (3a). Recrystallization from ethanol and water; violet solid; yield: 80\%; m.p.: $273-275{ }^{\circ} \mathrm{C}$; IR ( $\left.\mathrm{cm}^{-1}\right): 3099(\mathrm{~m}), 3053$ (w), 3007 (m), 1687 (s), 1629, 1613, 1579 (m), 1532 (m), 1451, 1374 (s); ${ }^{1} \mathrm{H}$ NMR $\left(250 \mathrm{MHz}, \mathrm{CD}_{2} \mathrm{Cl}_{2}\right): \delta 3.06\left(\mathrm{~s}, 6 \mathrm{H}, \mathrm{N}\left(\mathrm{CH}_{3}\right)_{2}\right), 3.28\left(\mathrm{~s}, 6 \mathrm{H}, \mathrm{N}-\mathrm{CH}_{3}\right), 6.70\left(\mathrm{~d},{ }^{3} J_{12,11}=\right.$ $9.0 \mathrm{~Hz}, 2 \mathrm{H}, 12), 6.81\left(\mathrm{dd},{ }^{3} J_{8,9}=15.2 \mathrm{~Hz},{ }^{3} J_{8,7}=10.4 \mathrm{~Hz}, 1 \mathrm{H}, 8\right), 7.24\left(\mathrm{~d},{ }^{3} J_{9,8}=15.2 \mathrm{~Hz}, 1 \mathrm{H}, 9\right)$, $7.44\left(\mathrm{~d},{ }^{3} J_{11,12}=9.0 \mathrm{~Hz}, 2 \mathrm{H}, 11\right), 9.44\left(\mathrm{~d},{ }^{3} J_{7,8}=10.4 \mathrm{~Hz}, 1 \mathrm{H}, 7\right) ;{ }^{13} \mathrm{C}\left\{{ }^{1} \mathrm{H}\right\}$ CP MAS NMR $(4$ $\mathrm{mm}, 12 \mathrm{kHz}): \delta 28.14,29.12,38.74,41.22,96.45,109.03,111.95,113.93,121.03,126.67$, $134.25,148.85,151.39,152.19,157.55,159.33$; anal. calculated for $\mathrm{C}_{17} \mathrm{H}_{20} \mathrm{~N}_{4} \mathrm{O}_{3}$ (found): $\mathrm{C}$ 62.18 (61.72), H 6.14 (6.00), N 17.06 (16.79).

1-Butyl-5-[(1E,2E)-3-\{4-(dimethylamino)phenyl\}prop-2-enyliden]aminobarbituric acid (3b). Recrystallization from ethanol and water; deep violet solid; yield: 64 \%; m.p.: $274-275^{\circ} \mathrm{C}$; IR (cm $\left.{ }^{-1}\right): 3157(\mathrm{~m}), 3030(\mathrm{~m}), 2949,2868(\mathrm{~m}), 1679,1652$ (s), 1582 (s), $1528(\mathrm{~m}), 1447(\mathrm{~s})$, $1370(\mathrm{~s}) ;{ }^{1} \mathrm{H}$ NMR $\left(250 \mathrm{MHz}, \mathrm{d}_{6}\right.$-DMSO): $\delta 0.88\left(\mathrm{t},{ }^{3} J_{4}, 3^{\prime}=7.3 \mathrm{~Hz}, 3 \mathrm{H}, \mathrm{CH}_{3}\right) 1.26(\mathrm{~m}, 2 \mathrm{H}$, 
$\left.\mathrm{CH}_{2}\right), 1.44\left(\mathrm{~m}, 2 \mathrm{H}, \mathrm{CH}_{2}\right), 2.99\left(\mathrm{~s}, 6 \mathrm{H}, \mathrm{N}\left(\mathrm{CH}_{3}\right)_{2}\right), 3.72\left(\mathrm{t},{ }^{3} J_{1^{\prime}, 2^{\prime}}=7.3 \mathrm{~Hz}, 2 \mathrm{H}, \mathrm{N}-\mathrm{CH}_{2}\right), 6.75(\mathrm{~d}$, $\left.{ }^{3} J_{12,11}=8.9 \mathrm{~Hz}, 2 \mathrm{H}, 12\right), 7.23-7.33(\mathrm{~m}, 1 \mathrm{H}, 8,9), 7.41\left(\mathrm{~d},{ }^{3} J_{11,12}=8.9 \mathrm{~Hz}, 2 \mathrm{H}, 11\right), 9.37-9.41(\mathrm{~m}$, $1 \mathrm{H}, 7), 10.34(\mathrm{~s}, 1 \mathrm{H}, 3) ;{ }^{13} \mathrm{C}\left\{{ }^{1} \mathrm{H}\right\}$ CP MAS NMR (4 mm, $\left.12 \mathrm{kHz}\right): \delta 15.51,21.51,33.36,38.74$, 40.59 , 95.85, 109.48, 113.11, 118.92, 124.45, 126.00, 132.50, 145.63, 148.09, 150.59, 152.76, 156.41, 159.24; anal. calculated for $\mathrm{C}_{19} \mathrm{H}_{24} \mathrm{~N}_{4} \mathrm{O}_{3}$ (found): $\mathrm{C} 64.02$ (63.46), H 6.79 (6.48), N 15.72 (15.55).

\section{Acknowledgements}

We are grateful to the University of Technology in Chemnitz for financial support. We thank A. Seifert, A. Mehner and R. Lungwitz for the solid state ${ }^{13} \mathrm{C}$ NMR spectroscopic measurements.

\section{References and Footnotes}

1. (a) Demeunynck, M.; Bailly, C.; Wilson, W. D. Eds., DNA and RNA Binders, Volume 2, Wiley-VCH: Weinheim, ISBN 3-527-30595-5, 2003.

2. (a) Bohanon, T. M.; Denzinger, S.; Fink, R.; Paulus, W.; Ringsdorf, H.; Weck, M. Angew. Chem. 1995, 107, 102; Angew. Chem. Int. Ed. 1995, 34, 58. (b) Bohanon, T. M.; Caruso, P.L.; Denzinger, S.; Fink, R.; Möbius, D.; Paulus, W.; Preece, J. A.; Ringsdorf, H.; Schollmeyer, D. Langmuir 1999, 15, 177.

3. (a) Würthner, F.; Yao, S.; Debaerdemaeker, T.; Wortmann, R. J. Am. Chem. Soc. 2002, 124, 9431. (b) Würthner, F.; Yao, S. J. Org. Chem. 2003, 68, 8943. (c) Würthner, F. W. Chem. Comm. 2004, 1564.

4. Voegel, J. J.; v. Krosigk, U.; Benner, S. A. J. Org. Chem. 1993, 58, 7542.

5. (a) Zimmerman, S. C.; Schmitt, P. J. Am Chem. Soc. 1995, 117, 10769. (b) Zimmerman, S. C.; Corbin, P. C. Structure and Bonding 2000, 96, 63.

6. Sherrington, D. C.; Taskinen, K. A. Chem. Soc. Rev. 2001, 30, 83.

7. Yu, L.; Schneider, H.-J. Eur. J. Org. Chem. 1999, 1619.

8. Sun, G.; Qiu, Y.-Q.; Sun, H. Z.;Su, Z.-M.; Feng, J.-D.; Zhu, Y. L. Chin. J. Chem. 2004, 22, 425.

9. Ivin, B. A.; Slesarev, V. I.; Smorygo, N. A.; Sochilin, E. G. Zh. Org. Khim. 1970, 6, 1326.

10. IR-data collection is available by the author (ina.bolz@chemie.tu-chemnitz.de).

11. Biltz, H.; Damm, P. Chem. Ber. 1913, 46, 3662.

12. Davidson, D.; Epstein, E. J. Org. Chem. 1936, 1, 305.

13. Stein, A.; Gregor, H. P.; Spoerri, P. J. Am. Chem. Soc. 1956, 78, 6185.

14. Irving, H.; da Silva, J. J. R. F. J. Chem. Soc. 1963, 458. 